\title{
Thermally Crosslinked Superparamagnetic Iron Oxide Nanoparticles: Synthesis and Application as a Dual Imaging Probe for Cancer in Vivo
}

Haerim Lee, ${ }^{\dagger}$ Mi Kyung Yu, ${ }^{\dagger}$ Sangjin Park,${ }^{\dagger}$ Sungmin Moon,${ }^{\ddagger}$ Jung Jun Min, ${ }^{\dagger}$ Yong Yeon Jeong, ${ }^{*}+\stackrel{\dagger}{*}$ Hae-Won Kang ${ }^{\xi}$ and Sangyong Jon $*, \dagger$

Characterizations of the carboxyl TCL-SPION. Synthesis of the poly(TMSMA-r-PEGMAr-NAS) was investigated by ${ }^{1} \mathrm{H}$ NMR spectra (JEOL JNM-LA 300 WB FT-NMR, Tokyo, Japan). FT-IR spectroscopy (SPECTRUM 2000 of PERKIN ELMER) was used to confirm the presence of the polymers in the carboxyl TCL-SPION. The saturation magnetization $(M \mathrm{~s})$ value of carboxyl TCL-SPION was measured by a magnetic property measurement system (MPMS) of Quantum Design at $300 \mathrm{~K}$. The applied magnetic field was varied from $10000 \mathrm{Oe}$ to -10000 Oe. Thermal gravimetric analysis (TGA) was carried out using TGA 2050 Thermogravimetric Analyzer (TA instruments). The temperature of the sample gradually increased from $40{ }^{\circ} \mathrm{C}$ to $600{ }^{\circ} \mathrm{C}$ at a rate of $10{ }^{\circ} \mathrm{C} / \mathrm{min}$.

Quantifying method on the number of Cy5.5 conjugated per TCL-SPION. Fluorescence intensity of Cy5.5 conjugated TCL-SPION solution was measured in a $1.0 \mathrm{~cm}$ path length cuvette (Hellma Quartz Glass Cell, 10x2 mm) by RF-5301PC spectrofluorophotometer of SHIMADZU. The total area for the fluorescence intensity $\left(\lambda_{\mathrm{ex}}=675 \mathrm{~nm}\right)$ was integrated from $685-740 \mathrm{~nm}$ with the excitation and emission slits set at $3 \mathrm{~nm}$. The intensity was changed into $\mu \mathrm{g}$ (nmol) Cy5.5 per mg TCL-SPION by the Cy5.5/D.W. standard calibration graph. 


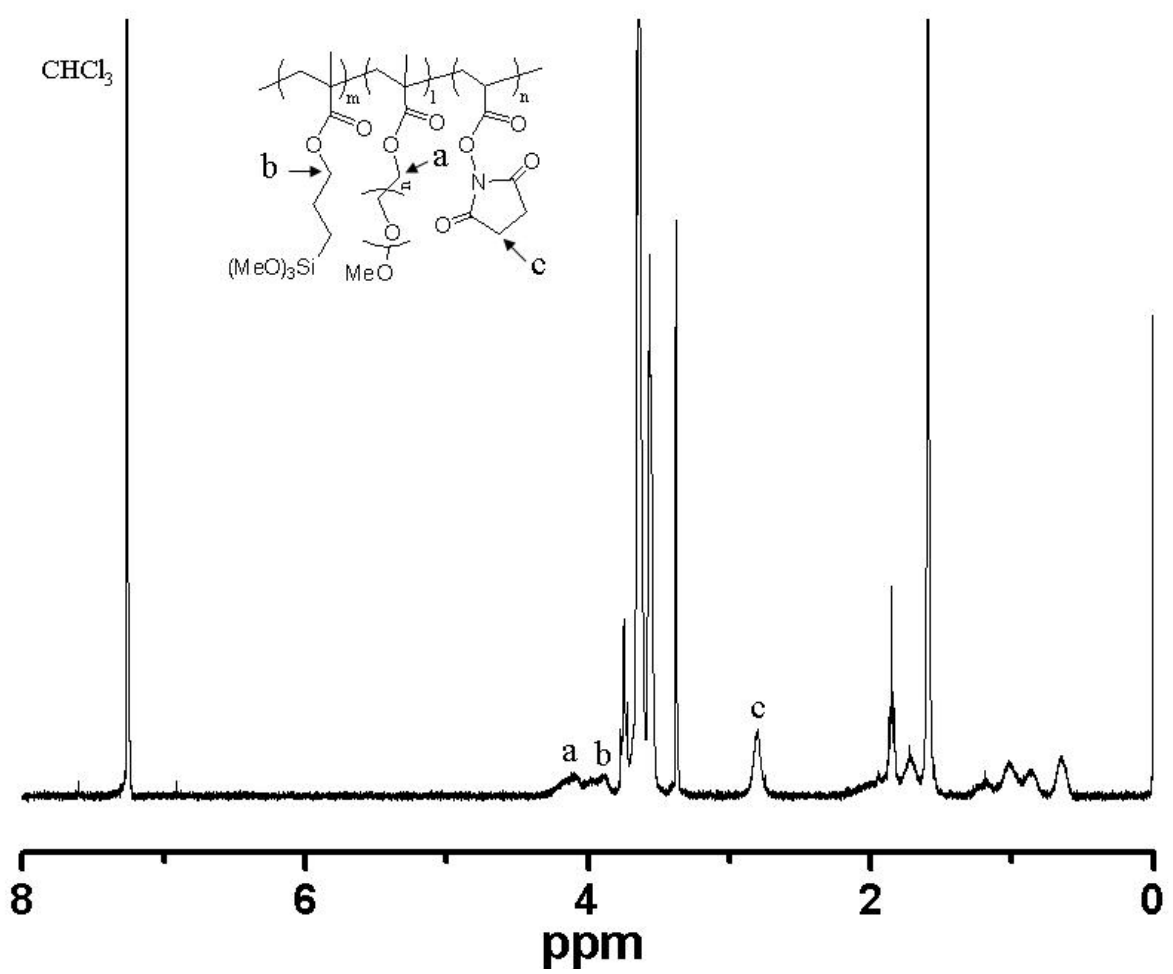

Figure S1. ${ }^{1} \mathrm{H}$ NMR spectra of the poly(TMSMA-r-PEGMA-r-NAS).

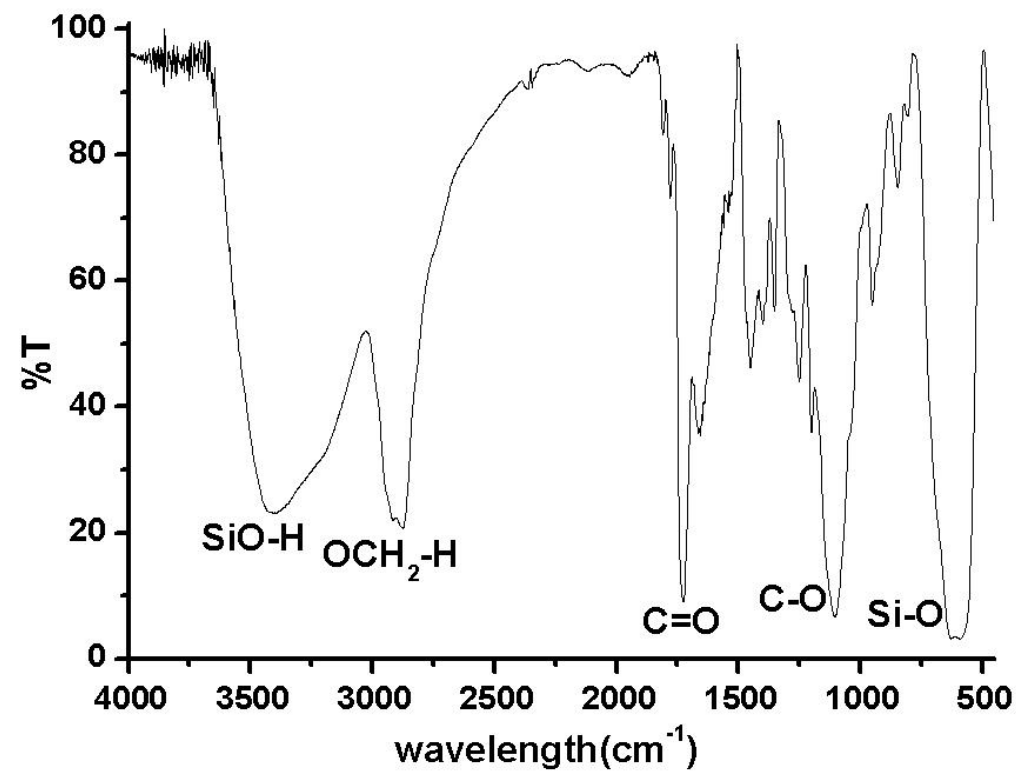

Figure S2. FT-IR spectra of the carboxyl TCL-SPION. 


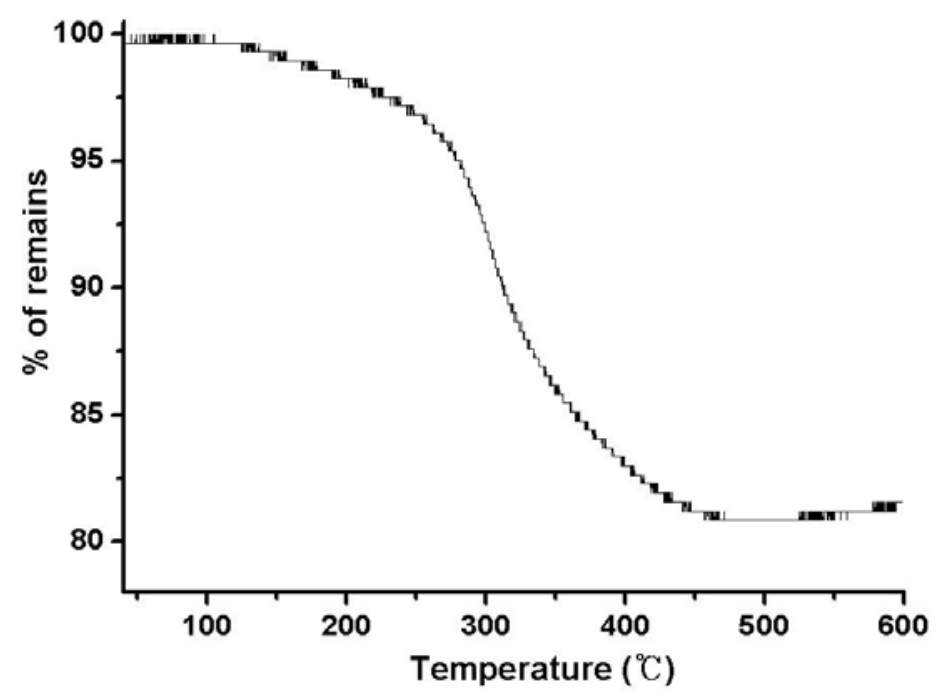

Figure S3. TGA graph of the carboxyl TCL-SPION.

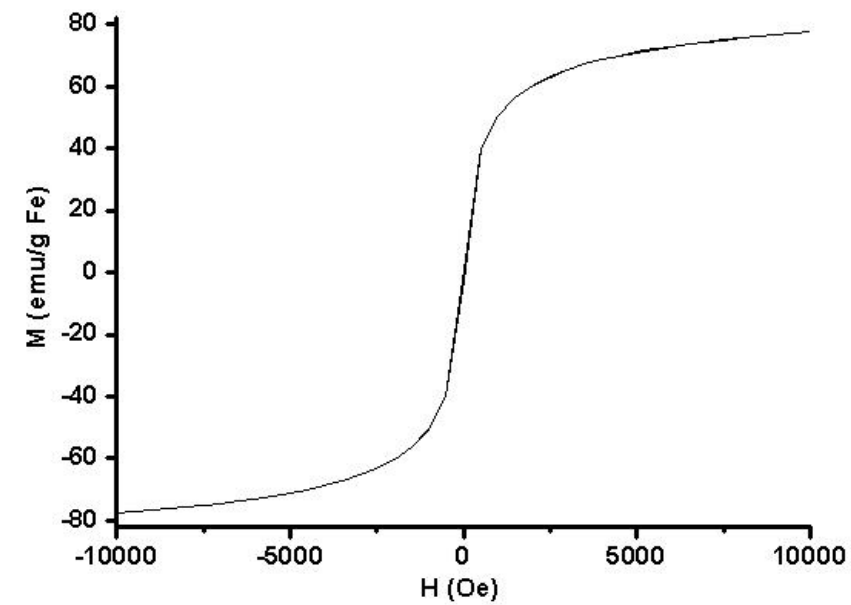

Figure S4. Variation of the magnetization of carboxyl TCL-SPION as a function of applied magnetic field. 


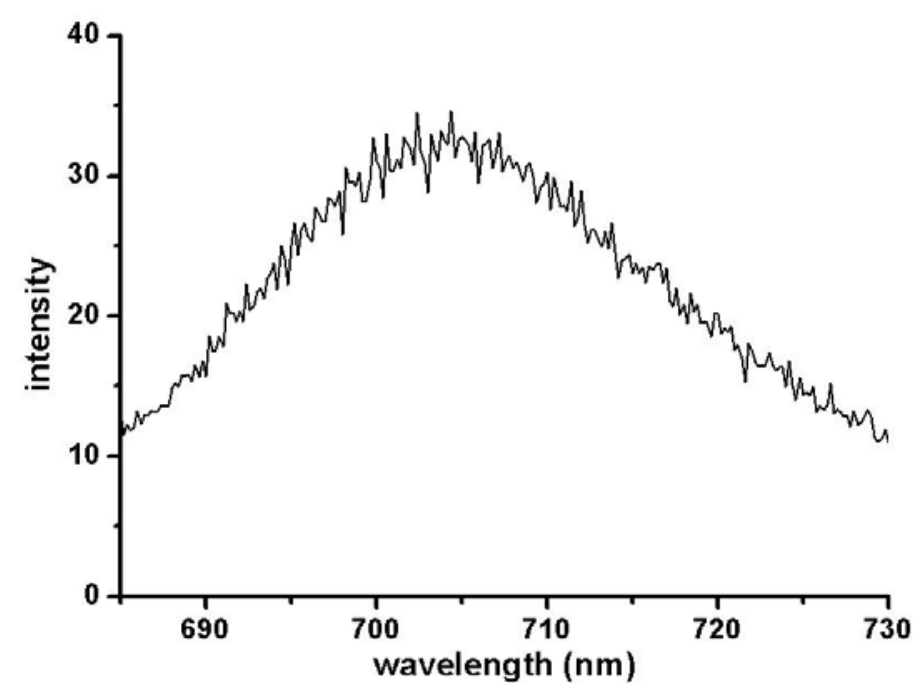

Figure S5. Fluorescence spectrum of the Cy5.5 TCL-SPION.

(a)
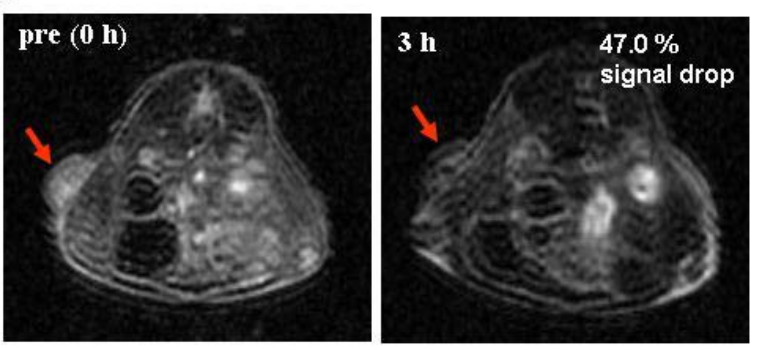

(b)

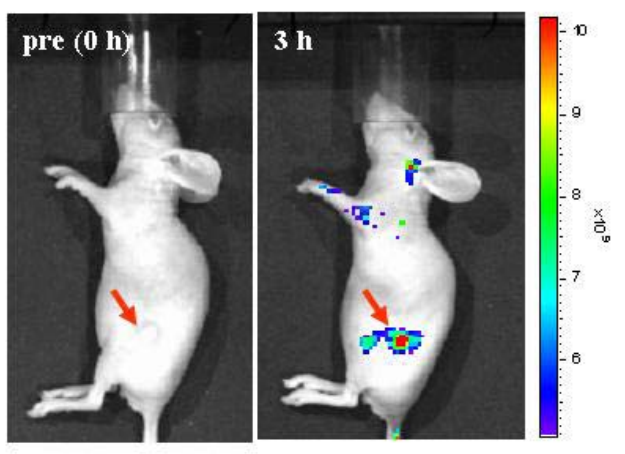

(c)

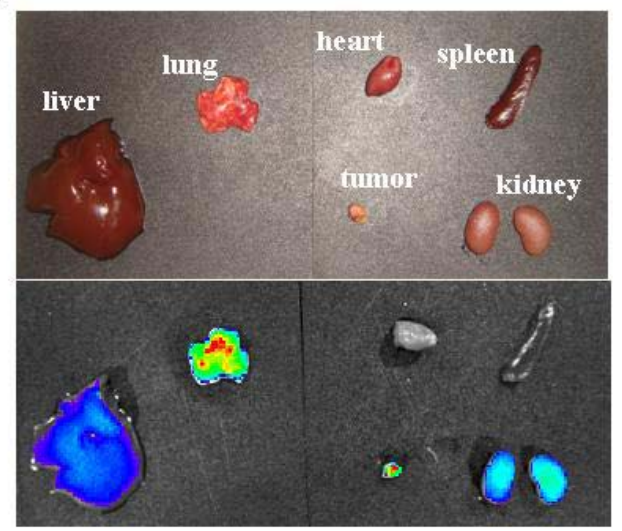

Figure S6. (a) T2-weighted fast-spin echo images (TR/TE of $4200 \mathrm{~ms} / 102 \mathrm{~ms}$ ) taken at $0 \mathrm{~h}$ and $3 \mathrm{~h}$ post-injection of $14.7 \mathrm{mg} \mathrm{Fe} / \mathrm{kg}$ of Cy5.5 TCL-SPION dispersed in 5\% glucose solution. (b) The whole body fluorescence images of the same mouse taken at $0 \mathrm{~h}$ and $3 \mathrm{~h}$ 
post-injection and (c) Photographic images and corresponding optical fluorescence images of several organs and the allograft tumor. Images were acquired with an exposure time of $1 \mathrm{~s}$ and with the Cy5.5 filter channel. The colored scale indicates the relative fluorescence intensity. The red arrows indicate the position of the allograft tumor.

(a)

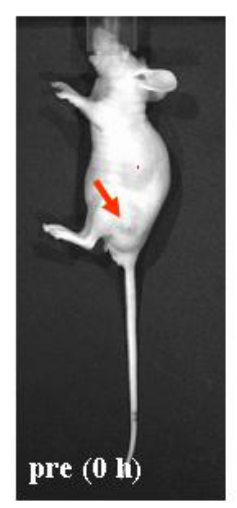

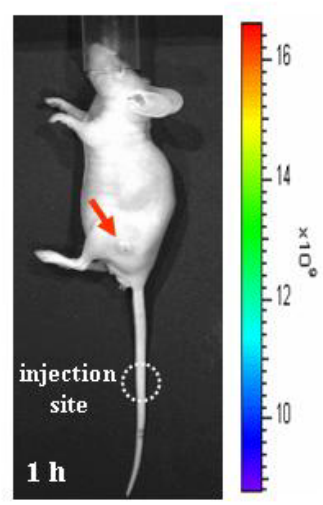

(b)

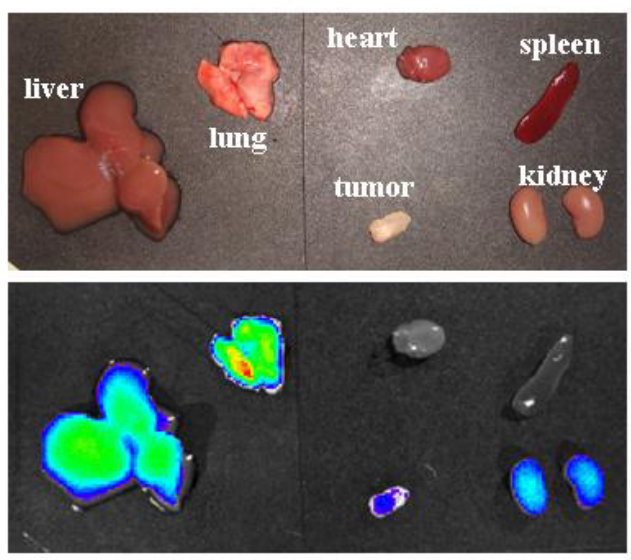

Figure S7. (a) The whole body fluorescence images of a tumor bearing mouse at $0 \mathrm{~h}$ and $1 \mathrm{~h}$ post-injection of a free Cy5.5 dye (the same amount as that used for Cy5.5 TCL-SPION of $14.7 \mathrm{mg} \mathrm{Fe} / \mathrm{kg}$ ). The red arrows indicate the position of the allograft tumor. (b) Photographic images and corresponding optical fluorescence images of several organs and the allograft tumor. Images were acquired with an exposure time of $1 \mathrm{~s}$ and with the Cy5.5 filter channel. The colored scale indicates the relative fluorescence intensity. 


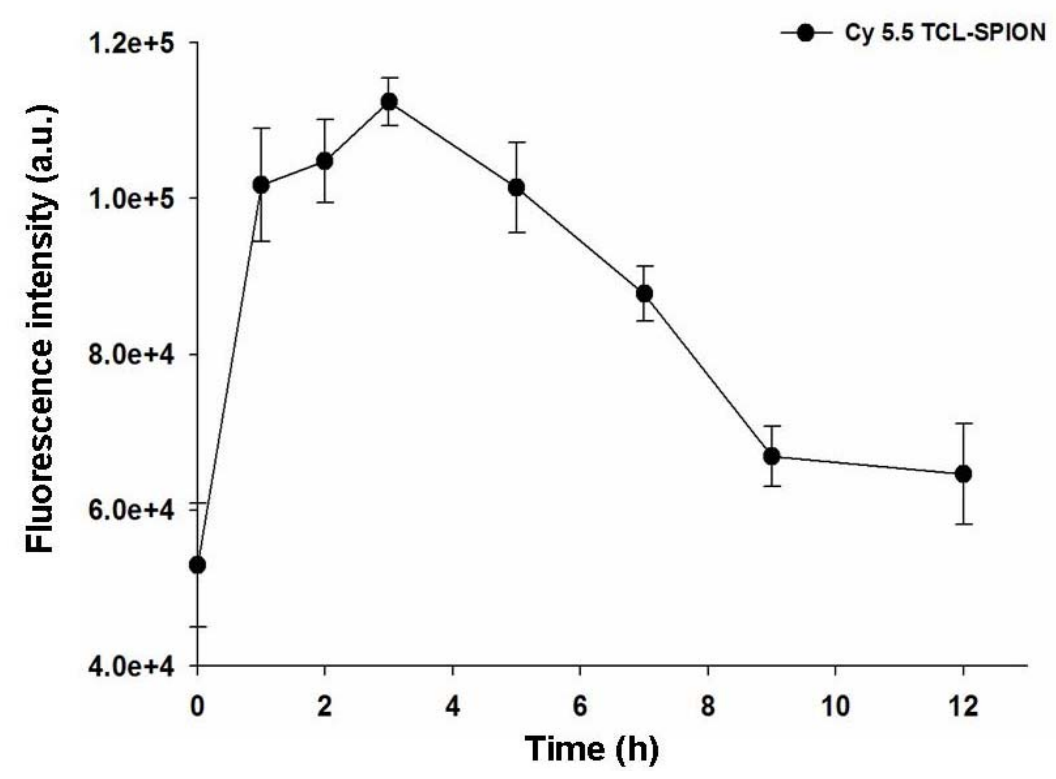

Figure S8. Mean Cy 5.5 TCL-SPION fluorescence intensity at the tumor areas as a function of time. After intravenous injection of Cy 5.5 TCL-SPION into tumor bearing mice $(n=3)$, fluorescence images were taken at indicated time $(0 \mathrm{~h}$ for pre injection and $1,2,3,5,7,9,12$ $\mathrm{h}$ for post injection, respectively). Three circular regions of interest (ROIs) values at the tumor areas were chosen to measure the relative fluorescence intensity. 


\section{Complete ref 5a, 10, 13, 14, 35b, 35c, and $35 f$}

5a. Lee, J. H.; Huh, Y. M.; Jun, Y. W.; Seo, J. W.; Jang, J. T.; Song, H. T.; Kim, S.; Cho, E. J.; Yoon, H. G.; Suh, J. S.; Cheon, J., Artificially engineered magnetic nanoparticles for ultrasensitive molecular imaging. Nat. Med. 2007, 13, 95-99.

10. de Vries, I. J.; Lesterhuis, W. J.; Barentsz, J. O.; Verdijk, P.; van Krieken, J. H.; Boerman, O. C.; Oyen, W. J.; Bonenkamp, J. J.; Boezeman, J. B.; Adema, G. J.; Bulte, J. W.; Scheenen, T. W.; Punt, C. J.; Heerschap, A.; Figdor, C. G. Nat. Biotechnol. 2005, 23, 1407-1413.

13. Nasongkla, N.; Bey, E.; Ren, J.; Ai, H.; Khemtong, C.; Guthi, J. S.; Chin, S. F.; Sherry, A. D.; Boothman, D. A.; Gao, J., Nano Lett. 2006, 6, 2427-2430.

14. Kim, J.; Park, S.; Lee, J. E.; Jin, S. M.; Lee, J. H.; Lee, I. S.; Yang, I.; Kim, J. S.; Kim, S. K.; Cho, M. H.; Hyeon, T. Angew. Chem. Int. Ed. 2006, 45, 7754-7758.

35b. Kim, S.; Lim, Y. T.; Soltesz, E. G.; De Grand, A. M.; Lee, J.; Nakayama, A.; Parker, J. A.; Mihaljevic, T.; Laurence, R. G.; Dor, D. M.; Cohn, L. H.; Bawendi, M. G.; Frangioni, J. V. Nat. Biotechnol. 2004, 22, 93-97.

35c. Veiseh, O.; Sun, C.; Gunn, J.; Kohler, N.; Gabikian, P.; Lee, D.; Bhattarai, N.; Ellenbogen, R.; Sze, R.; Hallahan, A.; Olson, J.; Zhang, M. Nano Lett. 2005, 5, 1003-1008.

35f. Orlova, A.; Magnusson, M.; Eriksson, T. L.; Nilsson, M.; Larsson, B.; HoidenGuthenberg, I.; Widstrom, C.; Carlsson, J.; Tolmachev, V.; Stahl, S.; Nilsson, F. Y., Tumor imaging using a picomolar affinity HER2 binding affibody molecule. Cancer Res. 2006, 66, 4339-4348. 